fournal of Medical Genetics (1976). 13, 80.

\section{Cerebral gigantism}

Sir,

I have read with interest the recent publication by Nevo and her colleagues (Nevo et al, 1974) which provides evidence for autosomal recessive inheritance of cerebral gigantism in an inbred family from Israel. The authors note that, while the possibility of genetic determination has been discussed by several authors, the evidence before their publication was insufficient and inconclusive.

In this respect we wish to draw attention to our publication (Townes and Scheiner, 1973) in which we described cerebral gigantism in three sibs (monozygotic twin girls and their brother) and concluded that the family provided clear evidence of autosomal recessive inheritance of this disorder. Though consanguinity was not proven in our family, it was considered possible since the parents were both born in a small village in Italy.

In their publication, Nevo et al (1974) conservatively conclude that: 'The family pattern leaves little doubt regarding the role of genetics in this particular case'namely, their family. Our observation in conjunction with theirs provides a reasonable basis for concluding that the autosomal recessive pattern of inheritance is probably not restricted to their kindred and may be of more general occurrence.

That our prior publication did not come to the attention of $\mathrm{Dr}$ Nevo and her co-workers is understandable since it was an abstract. However, it is important that the autosomal recessive inheritance of this disorder be recognized in order to increase the likelihood of appropriate genetic counselling. It is in this respect that we wish to draw attention to our earlier publication.

\section{Yours, etc,}

Philip L. Townes

Division of Genetics,

University of Rochester,

School of Medicine and Dentistry,

260 Crittenden Boulevard,

Rochester, New York 14642

REFERENCES

Nevo, S., Zeltzer, M., Benderly, A., and Levy, J. (1974). Evidence for autosomal recessive inheritance in cerebral gigantism. fournal of Medical Genetics, 11, 158-165.

Townes, P. L. and Scheiner, A. P. (1973). Cerebral gigantism (Sotos' syndrome): Evidence for recessive inheritance. (Abstract.) Pediatric Research, 7, 349.

\section{Diagnostic problems in cerebral gigantism} Sir,

Recently Nevo et al (1974) offered evidence for auto- $\vec{\circ}$ somal recessive inheritance in cerebral gigantism. They $\overrightarrow{\vec{H}}$ presented two affected sibships in a family with re- $\vec{\omega}$ current consanguineous matings $\left(F_{0}=\sum\left(\frac{1}{2}\right)^{n-1}\left(1+F_{A}\right)>\right.$ ? 0.1352 where $F_{o}$ is the inbreeding coefficient of the affected sibship, $F_{A}$ is the inbreeding coefficient of common. ancestor $\mathrm{A}$, and $\mathrm{n}$ equals the number of steps in the in- $\omega$ breeding loop). Thus, autosomal recessive inheritance $\overrightarrow{0}$ is clearly implicated and I have no quarrel with this.

On the other hand, the diagnosis of cerebral gigantismo is doubtful in my opinion. The patients reported by Nevo and her co-authors had (1) generalized oedema, (2) flexion contractures of the feet, (3) wrist drop with $\mathbb{D}$ clinodactyly, (4) extreme muscular hypotonia, (5) lowset malformed ears, and (6) other abnormalities. Although in many other respects they were similar to patients with cerebral gigantism, the overall pattern is at $\vec{\theta}$ variance with this diagnosis.

Defining the phenotypic spectrum from sporadic instances of a syndrome for which no biochemical test or chromosomal anomaly exists tends to truncate the syndrome population towards the severe end of the phenotypic spectrum. The more severe the abnormalities, the more likely they are to be ascertained. This is $\mathbb{Q}$ exactly how cerebral gigantism has been defined (all except three of the 80 previously reported cases have been $\overline{0}$ sporadic and no biochemical test or chromosomal anomaly is known). Therefore, striking features such as generalized oedema, flexion contractures, extreme mus-? cular hypotonia, clinodactyly, and low-set malformed ears would most likely have been reported previously if they were part of cerebral gigantism.

In my opinion, Nevo et al have probably described a new autosomal recessive overgrowth syndrome. The aetiology of cerebral gigantism is still unknown.

Yours, etc, M. Michael Cohen $\stackrel{\circ}{2}$

Departments of Orthodontics and Pediatrics, Schools of Dentistry and Medicine, University of Washington, Seattle, Washington, USA

\section{REFERENCE}

Nevo, S., Zeltzer, M., Benderly, A., and Levy, J. (1974). Evidenceo for autosomal recessive inheritance in cerebral gigantism. fournalc of Medical Genetics, 11, 158-165. 